\title{
RNA in Semen and Sperm: Importance and Transcriptome Analysis in Buck
}

\section{Saraswat $S^{*}$}

ICAR-Central Institute for Research on goats, India

Mini Review

Volume 3 Issue 2

Received Date: June 22, 2018

*Corresponding author: Sonia Saraswat, Scientist, APR division, ICAR-CIRG, Makhdoom, Farah, Mathura, UP-281122, India, Tel: 09997298248; Email: Published Date: July 27, 2018 mailto:sonia.saraswat@gmail.com

\section{Abstract}

Mammalian sperm contains an array of RNAs including messenger RNAs (mRNAs), ribosomal RNAs (rRNAs) and small RNAs (sRNAs), largely representing remnant transcripts produced during spermatogenesis. Increasing evidence has indicated that ncRNAs, which control gene expression at transcriptional, post-transcriptional, and epigenetic levels, play critical roles in male germ cell development. Sperm transcribe their RNA,RNAs have different role, high fertile buck have more RNA yield /spermatozoon vs low fertile buck, transcripts such as miR-34c, BMP2, TRADD influence semen quality and fertility.

Keywords: RNAs; Transcripts; Buck; Semen

\section{Introduction}

Reproductive success is crucial for species survival. Infertility is a disorder affecting humans as well as other animals. In caprine a number of bucks considered of highmerit based on their spermatozoa motility and morphology are unable to produce successful full-term pregnancies, according to extensive fertility data, suggesting that molecular defects affect the ability of spermatozoa to fertilize and contribute to normal embryo development. The integration of several tests, from standard procedures for the evaluation of sperm motility and viability, to sperm molecular investigation, is an approach to achieve a better understanding of sperm functions and to evaluate semen quality, predict buck fertility.
During fertilization, besides the paternal genome, spermatozoa transport coding and non coding RNAs into the oocyte. Mammalian sperm contains an array of RNAs including messenger RNAs (mRNAs), ribosomal RNAs (rRNAs) and small RNAs (sRNAs), largely representing remnant transcripts produced during spermatogenesis. RNA-Seq characterization of bovine spermatozoa revealed the presence of degraded and full-length nuclear-encoded transcripts involved incapacitation and fertilization, suggesting that RNA could be translated after spermatogenesis and potentially contribute to capacitation and early embryogenesis [1]. Furthermore, sperm transcripts retain information of the past events of spermatogenesis and contribute to egg fertilization and development. Sperm transcripts include sRNAs, are a class of short non-coding RNAs including different types of RNAs (i.e. microRNA (miRNA) and Piwi-interacting 
RNA (piRNA)), that play an essential regulatory role in spermatogenesis. Mammalian spermatogenesis begins with the self-renewal and differentiation of spermatogonial stem cells (SSCs). Spermatogonia differentiate to pre-leptotene spermatocytes by mitosis, and spermatocytes then undergo meiosis through the leptotene, zygotene, pachytene, and diplotene stages to produce haploid round spermatids. Round spermatids then undergo a dramatic differentiation process called spermiogenesis that includes acrosome formation, flagellum formation, nuclear condensation, and cytoplasmic exclusion to differentiate into elongated spermatids and eventually spermatozoa. Spermiogenesis in animals is a complex biochemical and morphological process consisting of at least 16 sequential transition steps [2]. In this review RNAs in semen in male goat is extensively being studied.

\section{miRNAs in Male Germ Cells}

miRNAs are derived from long primary transcripts that are synthesized predominantly by RNA polymerase II. These primary transcripts range in length from several hundreds to thousands of nucleotides (nts) and are termed primary miRNAs. In animals, primary miRNAs are initially processed into approximately $70 \mathrm{nt}$ precursor miRNAs (pre-miRNAs) by the RNase III member Drosha and its cofactor. Recent findings have indicated a general necessity for miRNAs in spermatogenesis, as genetic or siRNA-mediated ablation of key factors required for the biogenesis of miRNAs, including Dicer and Drosha, blocks male germ cell development. For instance, loss of Dicer in Sertoli cells severely impairs their competence and leads to progressive testicular degeneration, male infertility and a complete absence of spermatozoa, thereby suggesting an essential role of the Dicer-dependent miRNA/siRNA pathway in mammalian male germ cell development. Moreover, a recent study reported that selective inactivation of Drosha or Dicer in spermatogenic cells depletes spermatocytes and spermatids in the testes and leads to oligoteratozoospermia or azoospermia, thereby indicating that either the Drosha-dependent canonical miRNA pathway or the Dicer-dependent miRNA/siRNA pathway is critically important for male germ cell development. Importantly, miR-34c is expressed in SSCs of dairy goats and promotes apoptosis of SSCs in a p53-dependent manner [3], additionally, miR$34 \mathrm{c}$ is also present in mouse sperm, and this sperm-borne miRNA is important for the first cell division of zygotes via modulation of $\mathrm{Bcl}-2$ expression. Together, these findings suggest that a number of miRNAs are tightly regulated during spermatogonial differentiation, while these miRNAs play multiple roles in spermatogenic cells by regulating their specific targets [2].

Transition nuclear protein 2 (TNP2) and Protamine 2 (Prm2) are chromatin remodelers that are essential for spermatid elongation and completion of spermiogenesis. A recent study showed that testis-specific miR-469 sequestered Tnp2 and Prm2 mRNAs from translation in pachytene spermatocytes and round spermatids with a minor effect on their stability. In contrast, miR-122a, which is enriched in late-stage male germ cells and predominantly in polysomes, mediates the degradation of Tnp2 mRNA. Together, these findings indicate that miRNAs are involved in the post-transcriptional regulation of genes in spermatid development. Moreover, mir-23b, mir-30c, mir-30d, and mir-690 have been found to be regulated by FSH and androgens in Sertoli cells, and these miRNAs play important roles in cell adhesion and spermiation. In addition, the nucleus of mature spermatozoa contains a complex population of mRNAs and miRNAs, thereby suggesting potential functions in early embryonic development. Collectively, these findings demonstrate the importance of miRNA in the control of many functions, such as maintenance of spermatogonial stem cells (SSCs) status, regulation of SSCs differentiation, meitoic and post-meiotic processing and spermiogenesis. Dysregulation in miRNAs' expression patterns is severely affected in different types of reproduction abnormalities.

\section{siRNAs in Male Germ Cells}

siRNAs are a class of small RNAs that processed from double-stranded RNAs (dsRNAs) by Dicer, which are loaded onto Ago2 and mediate the cleavage of target mRNAs via the perfect or near-perfect base-pairing rule. This biological process has been termed as RNA interference (RNAi) and widely used as a tool to modulate gene expression in vitro and in vivo. In mammals, endosiRNAs has been first reported in murine oocytes and embryonic stem cells.

\section{piRNAs in Male Germ Cells}

The evolutionarily conserved piwi family genes, which encode the germline-specific members of the Argonaute protein family, are indispensable to germline development in animals.

\section{Isolation of Spermatozoa for mRNA}

The bucks has to be maintained under a semiintensive management system with 6-7 hours of grazing 
and stall feeding with seasonally available green fodder ad libitum, supplemented with concentrate mixtures depending upon the status and age category of the animals. The animals have to housed separately $(1.5 \mathrm{~km}$ apart) according to their ages, sex, physiological status and health status. Controlled breeding is too practiced with the does being bred during May-June and OctoberNovember followed by kidding in the months of OctoberNovember and March-April, respectively.

The bucks $(n=6)$ aged between 2 and 3 years, subjected to routine semen collection with the aid of the artificial vagina (AV). The volume of ejaculates was measured in a graduated conical collection cups at $0.1 \mathrm{ml}$ intervals and the mass activity (measured on 0-5 scale) was estimated using phase-contrast microscope (10x). The ejaculates quality parameters were studied viz. mass activity, volume, color, consistency and progressive motility. Ejaculates between 1 and $2 \mathrm{ml}$ in volume and with $>80 \%$ were included in this study. Further, ejaculates were diluted at $37^{\circ} \mathrm{C}$ in Tris-based extender (Tris $290 \mathrm{mM} / \mathrm{l}$, citric acid $100 \mathrm{mM} / \mathrm{l}$, fructose $100 \mathrm{mM} / \mathrm{l}$, glycerol 6\%) with $10 \%$ egg yolk [4-8]. Two groups of animals were divided on the basis of above seminal traits as G-I(having better seminal traits) and G-II(poor seminal traits). $1 \mathrm{ml}$ of whole semen from each ejaculatewas aliquoted in a 1.5-ml tube, snap-frozen in liquid nitrogen,and stored at $-80{ }^{\circ} \mathrm{C}$ until RNA isolation. Another 1 - $\mathrm{ml}$ aliquot ofsemen wascentrifuged at $5000 \mathrm{~g}$ for $5 \mathrm{~min}$ at $4{ }^{\circ} \mathrm{C}$, and after removingthe seminal plasma, the sperm pellet was snap-frozen in liquid nitrogenand stored at $80^{\circ} \mathrm{C}$ until RNA isolation by Trizol method. Further quantified and checked by gel electrophoresis. Furthermore, the first strand cDNA was synthesized from the isolated total RNA using Transcriptor first strand cDNA synthesis kit (Roche, USA) as per manufacturer's instructions. Real time polymerase chain reaction (RTPCR) was carried out using reverse transcription system (LC-480, Roche, USA). The quantitative RT-PCR was performed using Roche Applied Science SYBR Master Mix following manufactures instructions. The annealing temperature was standardized using cDNA prepared from mRNA by PCR. The reaction was carried out at different annealing temperatures, primer concentrations, $\mathrm{MgCl} 2$ concentration, template DNA and Taq polymerase.

\section{Conclusion}

In this study it is concluded that sperm transcribe their RNA, RNAs have different roles. High fertile buck has more RNA yield /spermatozoonvs low fertile buck, transcripts such as miR-34c, BMP2 and TRADD influence semen quality and fertility.

\section{References}

1. Capra E, Turri F, Lazzari B, Cremonesi P, Gliozzi TM, et al. (2017) Small RNA sequencing of cryopreserved semen from single bull revealed altered miRNAs and piRNAs expression between High- and Low-motile sperm populations. BMC Genomics 18: 14.

2. Gou LT, Dai P, Liu MF (2014) Small non coding RNAs and male infertility. WIREs RNA 5(6): 733-745.

3. Li M, Yu M, Liu C, Zhu H, He X, et al. (2013) miR-34c works downstream of p53 leading to dairy goat male germline stem-cell (mGSCs) apoptosis. Cell Prolif 46(2): 223-231.

4. Saraswat S, Jindal SK, Priyadharsini R, Ramachandran N, Yadav S, et al. (2012) The Effect of antioxidants supplementation to cryopreservation protocol on seminal attributes and sperm membrane characteristics in Sirohi goat. J Phys Pharm Adv 2(1): 49-58.

5. Saraswat S, Priyadharsini R, Jindal SK, Yadav S, Ramachandran N, et al. (2012) Effect of antioxidants supplementation at refrigeration temperature on sperm motion characteristics and membrane integrity of Sirohi buck Semen. J Phys Pharm Adv 2(1): 77-86

6. Saraswat S, Rout PK, Kharche SD, Jindal SK, Goel AK (2016) Molecular expression of caprine estrogen receptor gene 1 in reproductive and non reproductive tissues. Reproduction in Domestic Animals 51(6): 1049-1054.

7. Saraswat S (2016) Estrogen receptors-New players in spermatogenesis. Iranian Journal of Applied Animal Science 6(3): 503-504.

8. Saraswat S, Rout PK, Kharche SD, Jindal SK, Goel AK, et al. (2016) Estrogen receptor 1 gene expression in caprine and its effect on fertility. Iranian journal of Veterinary Research 17(3): 207-209.

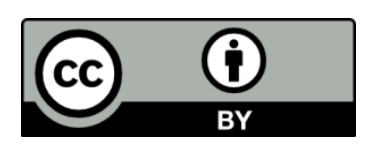

